\title{
DIPLEJIA FACIAL \\ UNA VARIANTE DEL SÍNDROME DE GUILLAIN BARRE
}

\author{
JULIANA VARGAS ${ }^{1 *}$, CARLOS NIEBLES ${ }^{2}$ \\ ${ }^{1}$ Servicio de Neurología, Hospital Militar Central. \\ Bogotá, Colombia \\ ${ }^{2}$ Neurólogo, Hospital Militar Central. Servicio de Neurología. \\ Bogotá. Colombia. \\ ${ }^{1 *}$ Correspondencia: ju_lianita@hotmail.com \\ Recibido: Marzo 4 de 2015 Aceptado: Julio 22 de 2015
}

\begin{abstract}
Resumen
El Síndrome de Guillain Barre es una polineuropatía autoinmune que causa desmielinización motora y sensitiva, frecuentemente con antecedente de una infección. El diagnóstico se realiza mediante sospecha clínica, aunque el líquido cefalorraquídeo y estudios electrodiagnósticos ayudan a soportarlo.

El Médico debe estar familiarizado con las variantes clínicas tales como la diplejía facial para hacer un diagnóstico preciso. La inmunoglobulina intravenosa y la plasmaferesis son tratamientos eficaces. El cuidado de soporte durante y después de la hospitalización es crucial.
\end{abstract}

Palabras clave: Diplejía facial, Guillain Barré, Inmunoglobulina, parálisis facial, variantes.

\section{FACIAL DIPLEGIA A VARIANT OF GUILLAIN BARRE SYNDROME}

\begin{abstract}
Guillain Barre Syndrome is an autoimmune polyneuropathy that causes motor and sensory demyelination, often with a history of infection. The diagnosis is made by clinical suspicion, although the cerebrospinal fluid and electrodiagnostic studies help support it.

The Physician should be familiar with the clinical variants such as facial diplegia and make an accurate diagnosis. IVIG and plasmapheresis are effective treatments. Supportive care during and after hospitalization is crucial.
\end{abstract}

Keys words: Facial diplegia. Guillain Barre, Inmunoglobulin, facial paralysis, variants. 


\title{
DIPLEGIA FACIAL UMA VARIANTE DA SÍNDROME DE GUILLAIN-BARRE
}

\begin{abstract}
Resumo
A síndrome de Guillain Barre é uma polineuropatia autoimune que provoca desmielinização motora e sensitiva, frequentemente com antecedentes de infecção. O diagnostico é realizado através de suspeita clinica, embora o líquido cefalorraquidiano e estudos eletrodiagnósticos ajudam a apoiá-lo.

O médico deve estar familiarizado com as variantes clínicas tais como a diplegia facial para estabelecer um diagnóstico preciso. A imunoglobulina intravenosa e a plasmaférese são tratamentos eficazes. $\mathrm{O}$ tratamento de suporte durante e após a hospitalização é crucial.
\end{abstract}

Palavras-chave: Diplegia facial, síndrome de Guillain Barre, imunoglobulinas, paralisia facial, variantes.

\section{Introducción}

Jean Baptiste Octave Landry en 1859 fue el primero en describir diez pacientes con debilidad distal ascendente después de pródromos de malestar general, fiebre, quién progreso a parálisis después de las tres semanas y muerte por falla respiratoria. Seis años después Georges Guillain, Jean-Alexandre Barre y Andre Strohl $(1,2)$ reportaron dos casos de soldados franceses con debilidad motora, arreflexia y disociación albumino citológica en líquido cefalorraquídeo.

El síntoma inicial común es la acroparestesia con leve pérdida objetiva de sensibilidad distal (3), asociado a severo dolor radicular o dolor neuropático. La mayoría de los pacientes presentan inicialmente debilidad en las piernas y brazos (32\%), debilidad proximal y distal en miembros inferiores (56\%), mientras que algunos tienen la debilidad que inicia en miembros superiores (12\%). La debilidad se presenta en las primeras dos semanas en el 50\% de los casos, y en las primeras 4 semanas en el 90\%. Una presentación descendente como en el botulismo, con inicio en cara y miembros superiores es menos común, hipo o arreflexia en los primeros días hasta la primera semana.

En 1953 Guillain descubrió formas de su enfermedad que afectaban el tronco cerebral, pero el trabajo final fue publicado por C Miller Fisher en 1956 que descubrió una nueva entidad con oftalmoplejia, ataxia y arreflexia sin debilidad en extremidades, los estudios neurofisiológicos sugieren un proceso axonal, con pequeños potenciales de acción sensorial sin disminuir las velocidades de conducción, los hallazgos en LCR (líquido cefalorraquídeo) son similares a Guillan Barre con buen pronóstico; en el año siguiente Bickerstaff describe una serie de casos con oftalmoplejia, signos piramidales, neuropatía y deterioro cognitivo, el LCR puede mostrar pleocitosis. Otras variantes (4) reconocidas fueron la faríngeo-cervico-braquial y la diplejía facial, en estas, los pares craneanos más afectados son IX, X y VII.

El nervio facial puede involucrarse en el síndrome del Guillain Barre en el 27\% al 50\% de los casos y la mitad de estos el compromiso es bilateral. La patofisiología que se encuentra en los nervios afectados es infiltración linfocítica celular o destrucción de la mielina (5).

La parálisis facial bilateral también denominada "Diplejía facial", es una condición poco común, que afecta 0.3 a $2 \%$ de todas las parálisis faciales, se afectan ambas hemicaras en un período de 4 semanas. Las causas más comunes son idiopáticas 20\% (6,7), síndrome Guillain Barre 10\%, neuropatías múltiples craneales, esclerosis múltiple, enfermedad de Parkinson, tumores de puente y meníngeos 21\%, infecciones (Borrelia, citomegalovirus, sífilis, VIH, herpes virus, mononucleosis, Epstein Barr, Mycoplasma pneumoniae, hepatitis A), causas congénitas (Síndrome de Moebius, miopatías), porfiria, sarcoidosis, lupus, fracturas del hueso temporal, amiloidosis, leucemia, linfoma, diabetes, alcoholismo. Esta entidad puede ser confundida con otras como distrofia facioescapulohumeral o miastenia grave, sin embargo esta última se asocia con ptosis palpebral, anormalidades en los movimientos oculares, no se asocian a parálisis del nervio facial (8-11).

\section{Descripción del paciente}

Reporte de un caso de un paciente que sufrió esta rara variante del Guillain Barre, el cual fue manejado exitosamente. 
Mujer de 44 años, empacadora de una empresa de plásticos, con cuadro de 6 semanas de evolución, que inició con síntomas gastrointestinales dados por deposiciones liquidas, dolor abdominal, vómito y fiebre por lo cual fue hospitalizada y recibió tratamiento antibiótico. Concomitante con lo anterior se inició proceso de dolor muscular generalizado de predominio lumbar, asociado a parestesias en manos bilateral. En la última semana presento parálisis facial periférica izquierda por lo que acudió a urgencias dieron manejo sintomático ambulatorio con Prednisona y Aciclovir; ingresa a urgencias por presentar aumento de su dolor muscular generalizado de predomino lumbar. Tiene antecedentes de Fibromialgia. Al examen neurológico de ingreso se encuentra limitación en el cierre ocular y signo de Bell positivo bilateral, prueba de ice eyes test no mejora el déficit, lagooftalmos bilateral, borramiento del surco nasogeniano bilateral, no se acompañó de hiperacusia, ni disgeusia; sin alteración motora, RMT (reflejos miotendinosos normales) normales, sin otros hallazgos positivos en el examen. Se decide hospitalizar para estudios de etiología de diplejía facial, se realiza punción lumbar, encontrando disociación albumino citológica, RNM (resonancia nuclear magnética) de columna dorsolumbar: Polirradiculitis de origen inflamatorio, RNM cerebral normal, Perfil infeccioso con Herpes simple, prueba de reagina plasmática rápida (RPR), bacilo ácido alcohol resistente (BAAR), Cultivo, Ig M varicela, Ac Epstein Barr, HIV negativos. Se consideró Síndrome de Guillain Barre variante diplejía facial, se hospitalizo en Unidad cuidado intensivo para inicio de Inmunoglobulina $G$ humana y vigilar patrón respiratorio, síndrome disautonómico; la paciente presento buena evolución sin presentar deterioro, no requirió ventilación mecánica, culminó 5 días de tratamiento con lo que se dio traslado a pisos, en donde continuó con su rehabilitación con un buen resultado clínico.

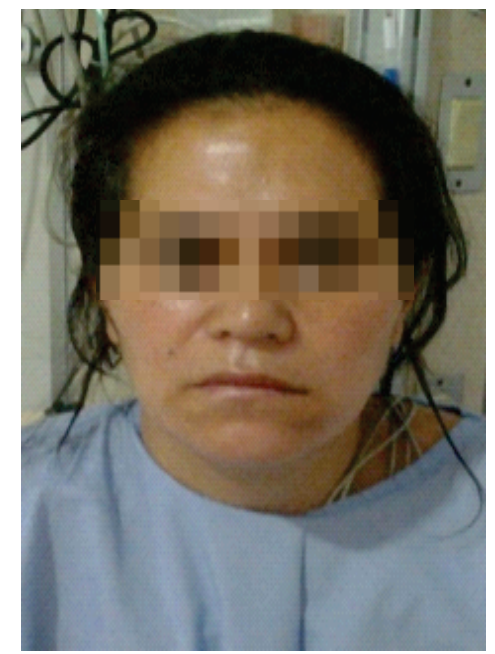

Figura 1. Paciente femenina de 44 años con borramiento de surcos nasogenianos bilateral.

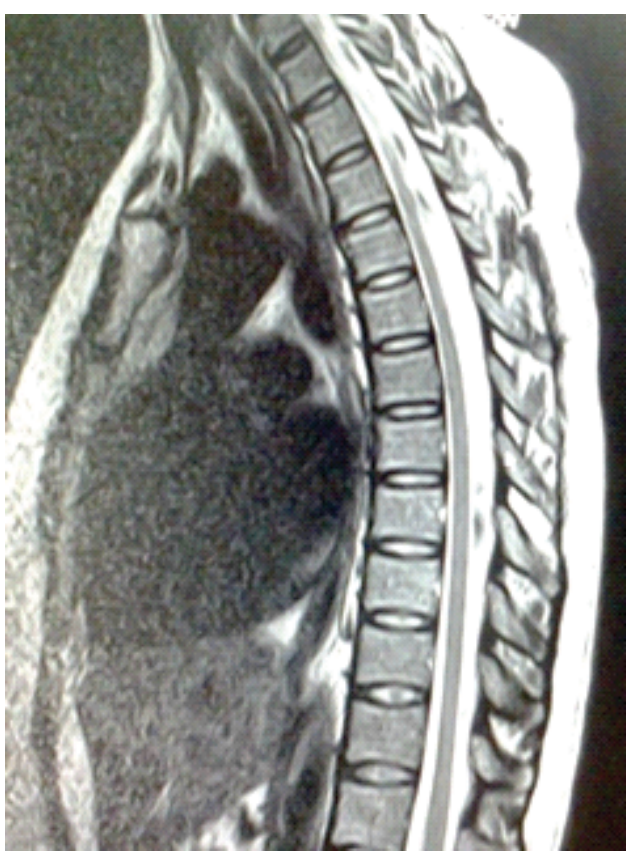

Figura 1. RMNmuestra poliradiculitis.

\section{Discusión}

La parálisis facial bilateral es una entidad rara pero representativa de enfermedades como esclerosis múltiple, sarcoidosis, infección, vasculitis, leucemia, enfermedad Lyme, mononucleosis, trauma, idiopática. La más relacionada es con Síndrome de Guillain Barre sin presentar debilidad muscular, sabiendo que esta se encuentra entre las variantes regionales: síndrome de Miller Fisher, debilidad faríngea cervico braquial $(12,13)$.La mayoría de pacientes presentan evolución favorable, particularmente cuando la inmunoglobulina o plasmaferesis es combinada con rehabilitación interdisciplinaria (14), con una recuperación hasta del $85 \%$ de su componente muscular facial y de las mialgias (15) como ocurrió en esta paciente. Similar a los pacientes con parálisis de Bell, donde el $71 \%$ recuperan a pesar de estar sin tratamiento (16) y un $84 \%$ recuperan su función normal (17).

En la serie de pacientes publicada por Susuki (18), en donde diagnosticaron 22 pacientes con diplejía facial, donde los síntomas iniciales, incluían parestesias que afectaban las extremidades, con parálisis facial bilateral que aparecían 3 a 10 días después, $86 \%$ tuvieron síntomas de infecciones en las últimas 4 semanas, encontrándose como prevalente anticuerpos citomegalovirus en un 35\% de los casos, en todos los pacientes se encontró disociación albumino citológica. En el reporte de caso publicado por Marzo Sola (19), los síntomas se presentaron posterior a la aplicación de 
la vacuna contra influenza A, el síntoma inicial fue dolor lumbar, con diplejía facial que la presento cinco días después, síntoma inicial no frecuente; en nuestro reporte de caso encontramos como síntoma inicial dolor lumbar. Se ha encontrado que la vacuna de la Influenza incrementa el riesgo de Guillain Barre (20), dos a siete semanas posteriores a la inmunización.

La asociación de diplejía facial e hiporreflexia debe hacer sospechar Guillain Barre, la disociación albumino citológica también soportan este diagnóstico. Los casos asociados con hiperreflexia, reportaron anticuerpos positivos Campylobacter jejuni.

En conclusión, la parálisis facial bilateral es una presentación infrecuente que se asocia en la mayoría de los casos a condiciones clínicas graves que pueden requerir inicio de tratamiento inmediato. El diagnóstico diferencial siempre debe incluir el Guillain Barre, en donde la disociación albumino citológica apoya el diagnóstico clínico. El manejo puede incluir soporte ventilatorio, infusión de inmunoglobulina o plasmaferesis. El pronóstico es bueno en la mayoría de pacientes tratados.

\section{Conflictos de interés}

Los autores declaramos no tener de manera directa o indirecta, ningún tipo de conflicto de intereses financieros, académicos o laborales que puedan poner en peligro la validez de este estudio.

\section{Financiación}

Este trabajo se realizó con el apoyo financiero de los investigadores.

\section{Referencias}

1. Landry O. Nore sur la paralysieascendanteaigue. Gaz Hebd Med Paris 1859;(6):472-4

2. Guillain G, Barré JA, Strohl A. Sur un syndrome de radilculone vriteavechyperalbuminose du liquidce phalorachidiensansreactioncellulaire. Remarques sur les catacteres cliniques graphiques de reflexesten dineux. Bull Mem Soc. Med. Hop Paris. 1996;40:1462-1470.

3. Barohn RJ, Saperstein DS. Guillain-Barre syndrome and chronic inflammatory demyelinating polyneuropathy. Semin. Neurol.1998;18:49-61.

4. Dididze MN. Clinical variants of Guillain-Barre syndrome: some aspects of differential diagnosis.G eorgian Med News . 2009; 166:48-51

5. Kilic R, Ozdek A, Felek S, Safak MA, Samim E. A Case Presentation of Bilateral Simultaneous Bell's Palsy. American Journal of Otolaryngology.2003;271-273.

6. Keane JR. Bilateral seventh palsy: analysis of 43 cases and review of the literature. Neurology. 1994;44:1198-1202.

7. Dimachkie MM, Barohn RJ. Guillain-Barre Syndrome and Variants. Neurol Clin. 2013;491-510

8. Susuki K, Koga M, et al. Guillain-Barre syndrome variant with prominent facial diplegia. J Neurol. 2009;256:1899-1905.

9. Sharma S, Kesav P. Bilateral facial nerve palsy: A rare association with hepatitis A. Ann Indian Academic Neurol. 2013;16:29-30.

10. Juntas R. Facial diplegia and acute inflammatory demyelinating neuropathy secondary to varicela. Rev Neurol (Paris) .2009;165(10): 836-838

11. Torres GM. Simultaneous bilateral facial palsy Acta Otorrinolaringol Esp 2009; 60(3):210-212

12. Stahl N, Ferit T: Recurrent bilateral peripheral facial palsy. J Laryngolotol 1989; 103(1):117-9.

13. Atsumi M, Kitaguchi M, et al .Ataxic Guillain-Barre syndrome with delayed facial diplegia. Rinsho Shinkeigaku 2003;43(9):548-551

14. Nitin K Sethi, Josh Torgounick, et al. Facial diplegia with hyperreflexia-a mild Guillain-Barre Syndrome variant, to treat or not to treat?. Journal of Brachial Plexus and Peripheral Nerve Injury. 2007;2:9.

15. Piñol G, Larrodér P, de la Puerta I, Tejero C, Iñiguez C, Santos S. Displejía facial: variante regional del síndrome de GuillainBarré. Ann Med Interna (Madrid). 2007;24(1):24-26.

16. Curtis CE, Barnes EV, Dupiche CA.-Barré syndrome variant: presenting with myalgias and acute facial diplegia. Mil Med. 2008;5:507-508.

17. Adour KK, Wingerd J. Idiopathic facial paralysis (Bell's palsy): factors affecting severity and outcome in 446 patients. Neurology. 1974;24:112-116.

18. Peitersen E. The natural history of Bell's palsy. Am J Otol 1982;4:107-111

19. Khlebtovsky A, Saban T, Steiner I. Unusual and benign course of idiopathic facial diplegia. Journal of Clinical Neuroscience.2013;20(6):904-905.

20. Marzo ME, M. Vaquero M, Bártulos M, Gil Á. Facial variant of Guillain-Barré Syndrome in a patient, days after being vaccinated for influenza A. Neurol. 2011;26(3):186-188. 